\title{
Polarization of the Recombination Line Maser in MWC349
}

\author{
C. Thum $^{1}$, D. Morris ${ }^{2}$, and H. Wiesemeyer \\ ${ }^{1}$ IRAM, Avenida Divina Pastora, 7, \\ Núcleo Central, E 18012 Granada, Spain \\ email: thum@iram.es \\ ${ }^{2}$ IRAM, 300 rue de la Piscine, \\ Domaine Universitaire de Grenoble \\ 38406 Saint Martin d'Hres, France \\ email: morris@iram.fr \\ ${ }^{3}$ MPIfR, Auf dem Hügel 51 \\ 5300 Bonn, Germany \\ email: wiesemeyer@mpifr.mpi-bonn.de
}

\begin{abstract}
We present observations of the circular polarization of the recombination line maser in MWC 349. Six good quality H30 $\alpha$ spectra were obtained during $2010-2011$ which show that the Zeeman features are complex, time variable, and usually different for the blue- and red-shifted maser spikes. We propose that the magnetic field, located in the corona of the circumstellar disk, has toroidal and radial components. It is plausibly generated in a disk dynamo.
\end{abstract}

Keywords. stars: individual (MWC 349), stars: magnetic fields, stars: winds, outflows

\section{Introduction}

The optically inconspicuous peculiar emission line star MWC 349 is one of the brightest radio stars and a strong emitter in the mid infrared. Its spectral energy distribution (Fig. 1) has two components: (i) the emission from hot circumstellar dust peaking near $\lambda=10 \mu \mathrm{m}$ and (ii) the emission from an ionized wind which follows a $\nu^{0.7}$ power law from $\lambda \lesssim 350 \mu \mathrm{m}$ to $\gtrsim 21 \mathrm{~cm}$. Radio recombination lines emitted by the wind at $\mathrm{cm}$ wavelengths were detected by Altenhoff et al. (1981). Observations at millimeter wavelengths reveiled that the recombination lines at wavelengths shorter than $3 \mathrm{~mm}$ are masing (Martín-Pintado et al. 1989). Subsequent investigations (Thum et al. 1998) showed that the amplification peaks near $\lambda=300 \mu \mathrm{m}$ and terminates at quantum number $n=7$ at $19 \mu \mathrm{m}$.

The blue- and red-shifted maser spikes (Fig. 2) mark the radial velocities where maser amplification is strongest. These spikes are located symmetrically about the star in the plane of the circumstellar disk which is seen nearly edge-on (Martín-Pintado et al. 2011). These investigators measure the radial distance of the spikes from the star as 29 a.u. The strong magnetic field found by Thum \& Morris (1999) from Zeeman features in the H30 $\alpha$ spectrum is therefore located at this large distance from the star in the ionized corona of the circumstellar disk.

In the present contribution, we report new polarization observations of the H30 $\alpha$ transition obtained with much improved instrumentation. The new data help to characterize the origin of the magnetic field and shed new light on the physical nature of the star. 


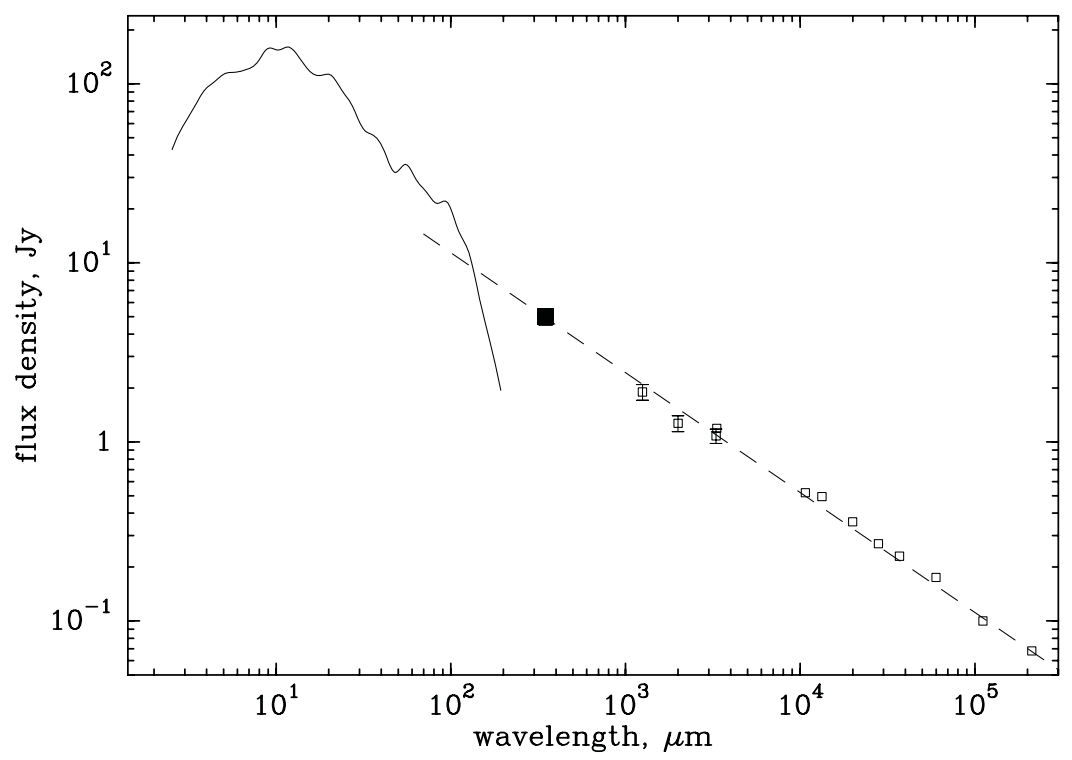

Figure 1. Spectral energy distribution of MWC 349. The continuous line is derived from ISO observations where the numerous emission lines have been subtracted. The dashed line is a fit to radio $/ \mathrm{mm} / \mathrm{submm}$ observations (Altenhoff et al. 1994; Tafoya et al. 2004). The $350 \mu \mathrm{m}$ observation (filled square) was made by Weiss \& Thum (unpubl.) at APEX.

\section{Observations}

Using the observing procedure XPOL at the IRAM 30m telescope (Thum et al. 2008) we monitored the $\mathrm{H} 30 \alpha$ line at $231.9 \mathrm{GHz}$ in all Stokes parameters during the period of April 2010 to January 2012. This transition was selected because its frequency is high enough for the maser to have strong amplification, while it is still easily accessible from the $30 \mathrm{~m}$ telescope. Altogether, six spectra of sufficient quality were obtained. All spectra were observed with identical instrumental setup. The spectral backend was set to a resolution of $80 \mathrm{kHz}\left(0.1 \mathrm{~km} \mathrm{~s}^{-1}\right)$.

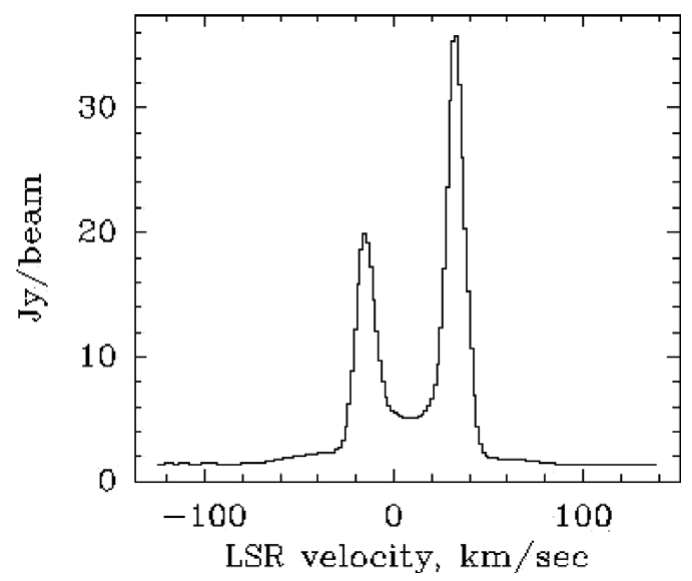

Figure 2. H30 $\alpha$ spectrum of MWC 349 obtained with the IRAM $30 \mathrm{~m}$ telescope at $231.9 \mathrm{GHz}$ (Stokes $I)$. The blue- $\left(\mathrm{v}_{L S R}=-18 \mathrm{~km} \mathrm{~s}^{-1}\right)$ and red-shifted $\left(\mathrm{v}_{L S R}=+32 \mathrm{~km} \mathrm{~s}^{-1}\right)$ maser spikes are superposed on a broad pedestal. The baseline offset of $1.7 \mathrm{Jy}$ is due to free-free continuum emission of the stellar wind. 
In this setup, the most important effects causing instrumental polarization are misalignment, phase errors, and the beam squint. Misalignment between the horizontal and vertical receiver channels of EMIR is small and stable (Carter et al. 2012). It causes a slighly elevation dependent instrumental Stokes $\mathrm{V}$ of the order of $V_{i}=0.2 \%$, as derived from observations of unpolarized point sources. Very similar values of $V_{i}$ are obtained when subtracting from the V- spectra of MWC 349 a fraction of the Stokes I spectrum so that the net power on the $\mathrm{V}$-spectrum is zero (positive and negative features are equally strong).

Errors of the phase between the two receiver channels transport power from Stokes $U$ into $V$, and could thus cause false $V$ signals. This effect can however be neglected, since the phase drift of XPOL is smaller than $1^{\circ}$ per hour and the source is not linearly polarized. We also ignore beam squint which operates only on extended sources.

The observed $V$-spectra were thus corrected for the misalignment error. They were then normalized such that the negative velocity half $\left(v_{L S R}<v_{s}\right.$, where $v_{s}=8.2 \mathrm{~km} \mathrm{~s}^{-1}$ is the systemic velocity) of the spectra was devided by their peak power of the blue-shifted maser spike, and their positive velocity part by the peak power of the red-shifted spike. The resulting spectra are free of systematics down to a level of $\sim 0.1 \%$. They are shown in Fig. 3, together with the original spectrum obtained by Thum \& Morris (1999).

\section{Results}

The observed spectra clearly show spectral features similar to those already detected in 1999 by Thum \& Morris. As argued by these authors, the observed features are best interpreted as due to the Zeeman effect. Although maser propagation effects can in principle generate Zeeman-like features (Nedoluha \& Watson 1994), the special conditions needed for these effects to operate are not met in the case of the recombination line maser.

The time series of spectra (Fig. 3) clearly shows that the observed Zeeman features are time variable. Within the limits given by our rather incomplete sampling, we conclude that the typical time scale of variation is much less than a year and larger than a few days. The time scale of $\mathrm{B}$-field variations is therefore similar to the time scale of total power variations of the $\mathrm{H} 30 \alpha$ maser which was found to be $\sim 1$ month (Thum et al. 1992).

Further inspection of the spectra gathered in Fig. 3 shows that the blue- and red-shifted spectral features are mostly very different from the standard antisymmetric $(S$-shaped) Zeeman signature. Often, we observe rather symmetric $(W$-shaped) features. Whereas $S$ shaped features are produced by homogenous fields, $W$-shaped features require that the maser propagates in a medium where the velocity has gradient and where the magnetic field reverses its direction. Both conditions are easily met in the corona of a rotating disk. As a consequence of the edge-on viewing geometry, the observed line-of-sight component of the field translates into a toroidal disk field. The occurance of $W$-shaped features implies however that there are also radial components present.

The strength of the field is estimated from the approximately $S$-shaped features to be about 20-25 mG. This value refers however only to the line-of-sight component. The total field strength can only be higher. Such a field must be considered strong, both in comparison with fields reported for molecular cores (e.g. Myers \& Goodman 1994) as well as compared with the thermal energy density of the plasma where the maser propagates. We estimate the plasma $\beta$-coefficient to be $\gtrsim 0.7$ which makes the observed field dynamically important. 




Figure 3. Time sequence of circular polarization spectra obtained with the IRAM $30 \mathrm{~m}$ telescope. The transition observed is $\mathrm{H} 30 \alpha$ at $231.9 \mathrm{GHz}$. Spectra were smoothed to a resolution of $0.4 \mathrm{~km} \mathrm{~s}^{-1}$. The vertical dashed lines indicate the peak velocities of the blue- and red-shifted maser spikes.

\section{Discussion}

The observed polarization characteritics suggest that the magnetic field associated with MWC 349 is very complex. The field strength varies on the time scales of order one month, orders of magnitude faster than the disk rotation period ( $\sim 35$ years). The field probed by the blue- and red-shifted spikes which are located symmetrically about the star seem to vary independently from each other, both in strength and orientation. 
We suggest on the basis of these characteristics that the field is generated locally in the dense disk corona by a dynamo process. Such a dynamo has been proposed by Tout \& Pringle (1992).

\section{References}

Altenhoff, W. J., Strittmatter, P. A. \& Wendker, H. J. 1981, A\&AA, 93, 48

Altenhoff, W. J., Thum, C., \& Wendker, H. J. 1994, A\&A, 281, 161

Carter, M., Lazareff, B., Maier, D., et al. 2012, A $6 A$, 538, A89

Martín-Pintado, J., Bachiller, R., Thum, C., \& Walmsley, C. M. 1989, A\&\&A, 215, L13

Martín-Pintado, J., Thum, C., Planesas, P., \& Báez-Rubio, A., 2011 A\&3A, 53, L15

Myers, P. C. \& Goodman, A. A. 1988, ApJ, 326, L27

Nedoluha G. E. \& Watson W. D. 1994, ApJ, 423, 394

Tafoya, D., Gómez, Y., \& Rodríguez, L. F. 2004, ApJ, 610, 827

Thum C., Martín-Pintado J., \& Bachiller R. 1992, A\&A, 256, 507

Thum, C., Martín-Pintado, J., Quirrenbach, A., \& Matthews, H. E. 1998, A\&3A, 333, L63 - L66

Thum, C. \& Morris, D. 1999 A\&A, 344, 923 - 929

Thum, C., Wiesemeyer, H., Paubert, G., Navarro, S., \& Morris, D. 2008, PASP, 120, 777

Tout C. A. \& Pringle J. E. 1992, MNRAS, 259, 604

White, R. L. \& Becker, R. H. 1985, ApJ, 297, 677 